\title{
CAUSAS DE ABANDONO DE LA PRÁCTICA CLÍNICA HOSPITALARIA DE ENFERMERÍA
}

\section{CAUSES OF HOSPITAL CLINICAL PRACTICE DROPOUT IN NURSING}

\author{
Alejandro Trapp U. \\ ANa I. LaRrain S.* \\ M. Jimena SANTis E. ${ }^{* * *}$ \\ SONIA OlbRich G. ${ }^{* * *}$
}

\begin{abstract}
RESUMEN
En los centros hospitalarios de Chile se observa la tendencia en las enfermeras de abandonar la práctica clínica hospitalaria tempranamente. Esto afecta el cuidado del paciente. Objetivo: Determinar las variables asociadas al abandono de las enfermeras de la práctica clínica hospitalaria $(\mathrm{PCH})$ en la Región Metropolitana. Material y método: A 1.000 enfermeras de la Región Metropolitana se enviaron encuestas vía e-mail y se obtuvieron 164 respuestas de enfermeras que abandonaron y 75 de enfermeras que no han abandonado la práctica hospitalaria. La encuesta consideraba variables asociadas a insatisfacción laboral como causas de abandono recogidas en la literatura y de un focus group. Resultados: Las principales causas de abandono fueron: el sistema de turnos, las remuneraciones y el escaso reconocimiento organizacional. La vocación aparece como la principal razón de permanencia en la práctica clínica. Conclusiones: El modelo predictivo propuesto permite pronosticar la probabilidad de que una enfermera abandone la práctica clínica. La insatisfacción con elementos organizacionales lleva al abandono. Este estudio puede ser un aporte al management en salud.
\end{abstract}

Palabras clave: Recursos humanos, personal de enfermería en hospital, satisfacción laboral, investigación en administración de enfermería.

\begin{abstract}
In Chilean hospitals nurses tend to drop out of their hospital clinical practice at an early stage of their professional life. This affects the patient care. Objective: to define the variables associated with nurses resignation to clinical practice in the Region Metropolitana (Metropolitan Region). Materials and method: 1,000 surveys were sent via e- mail to nurses in the Metropolitan Region of Chile, 164 responses were obtained from nurses who had left their clinical practice and 75 from nurses who were still in clinical practice. Results: The main causes of nurses' dropout from clinical practice were: the shift system, wages and weak organizational recognition. Regarding retention factors in clinical practice, vocation appears as the main reason. Conclusions: The proposed preliminary predictive model allows predicting the probability of a nurse leaving their clinical practice. Dissatisfaction with organizational elements leads to dropping out. This study may be a contribution to health management.
\end{abstract}

Key words: Human resources, nursing staff, hospital, job satisfaction, nursing administration research.

Fecha recepción: 31/01/2014 Fecha aceptación: 06/04/2015

\footnotetext{
* Magíster en Estadística. Profesor Asociado Escuela de Psicología, Universidad de los Andes. Chile. E-mail: atrapp@uandes.cl ${ }^{* *}$ Enfermera, Profesor Titular Escuela de Enfermería Universidad de los Andes. Chile. E-mail: alarrain@uandes.cl

${ }^{* * *}$ Enfermera, Magíster en Administración de Empresas, Universidad de Chile. Chile. E-mail: atrapp@uandes.cl

${ }^{* * * *}$ Psicóloga, Magíster en Dirección Estratégica de Recursos Humanos, Universidad Adolfo Ibáñez. Chile. E-mail: atrapp@ uandes.cl
} 


\section{INTRODUCCIÓN}

La Organización para la Cooperación y el Desarrollo Económicos (OCDE) informa que el estándar promedio de enfermeras por habitante es de 8.8 profesionales por cada 1.000 habitantes. Este número, en el 2013, era en Chile de 5.6, de 2.6 en México, 11.6 en Canadá y 5.54 en España (1), aun cuando la evidencia apoya que con una mayor proporción de enfermeras por paciente se logra una alta calidad de cuidados, se disminuyen las tasas de eventos adversos y la mortalidad (2-5). Para los sistemas de salud es difícil mantener óptimos indicadores al respecto y la constatación empírica de los autores de esta investigación permite señalar que es común, entre los directivos de enfermería de los centros hospitalarios de Chile, la percepción de una tendencia de las enfermeras de atención directa al paciente, a abandonar su trabajo luego de algunos años, migrando hacia cargos administrativos, hacia otras áreas de la enfermería e incluso hacia la asistencia en industrias. Este éxodo de enfermeras clínicas, sumado a un aumento en la demanda por atención, provoca un déficit de enfermeras expertas disponibles para la atención directa de los pacientes, las que son reemplazadas por profesionales de menor experiencia, frecuentemente recién egresadas. Esta situación afecta el ejercicio del cuidado de enfermería, pues se pierde capital de conocimiento experimentado y disminuye la posibilidad de traspaso intergeneracional desde las enfermeras expertas a las nóveles, con sus consecuencias sobre la calidad y seguridad de la atención profesional prestada.

La literatura a nivel mundial permite observar aquellas variables más relevantes que explicarían, no solo este abandono, sino también los fenómenos de rotación del recurso humano. Simon, Müller y Hasselhorn (6) observaron que la intención de abandonar la profesión era más frecuente que la intención de abandonar la organización y esto se rela- cionaba muy fuertemente con variables no vinculadas al trabajo, es decir, con factores asociados a antecedentes personales y de la interfaz trabajo-hogar. La intención de abandono de la organización se relacionaba con liderazgo organizacional y el contexto local, por lo que concluyeron que las políticas de retención de la enfermera en la práctica de la profesión debían dirigirse a esa interfaz. Por su parte, Black, Spetz y Harrington (7) compararon a un grupo de enfermeras que trabajaban fuera de la profesión con aquellas que aún estaban en ejercicio para determinar si esos dos grupos eran sustantivamente diferentes en relación a variables sociodemográficas, de mercado y políticas. El perfil diferenciado por sexo de los que dejaban la profesión para trabajar en otros ámbitos fue el siguiente: los enfermeros, eran solteros o casados y las enfermeras eran bachilleres, casadas, con hijos menores de 6 años, con alto ingreso familiar y mayor cantidad de años de graduadas. Los sueldos resultaron ser predictores de abandono de la profesión para las enfermeras solteras, pero no así para las casadas $(7,8)$.

Otras investigaciones han concluido que la satisfacción laboral y el compromiso organizacional son antecedentes clave para evaluar la rotación laboral, pues ambos fenómenos predicen la intención de cambiarse de trabajo (9) y aquellas enfermeras cuya intención de cambiarse es importante, son más jóvenes y con una menor trayectoria laboral $(10,11)$.

Shacklock y Brunetto (12) identificaron, en enfermeras de distintas generaciones, 6 variables que influían en la intención de abandonar la profesión: conflicto familiatrabajo, percepción de autonomía, apego al trabajo, importancia del trabajo para la persona, relación supervisor-subalterno y relaciones interpersonales. Sólo el apego al trabajo resultó ser una variable predictora de la intención de permanecer en la práctica hospitalaria en todas las generaciones (12). Reitz y Anderson (13) utilizaron el concepto "job embeddedness", que se podría traducir 
como "apego al trabajo" (AT), para explicar la rotación en las enfermeras. Encontraron que existía una relación inversa entre AT y la intención de desertar, y en algunos casos con la rotación efectiva. Liu et al. (14) observaron que la satisfacción laboral fue el predictor más significativo de permanencia en el trabajo. También identificaron que la edad, la experiencia laboral y el estado civil de casada fueron predictores significativos de la intención de no abandonar el trabajo. Respecto de la edad, observaron que las enfermeras por sobre los 34 años de edad tendían a quedarse y las con más de 5 años de experiencia estaban más dispuestas a permanecer en el trabajo. Tourangeau et al. (15) observaron que las buenas relaciones laborales, una percepción clara de logros personales y un menor desgaste emocional, aumentaban los niveles de satisfacción laboral y disminuyen la intención de abandonar el trabajo (15). Por su parte, Ulrich et al. (16) observaron que las jefaturas o los líderes de enfermería tenían un papel clave en la disminución del abandono y Choi et al. (17) concluyeron que las buenas relaciones laborales, el reconocimiento y el desarrollo profesional, eran factores críticos para evitar el abandono en los hospitales públicos de Hong Kong. Así mismo observaron que las enfermeras expresaban frustración e intención de dejar el ambiente laboral cuando este era desfavorable $(18)$. Diversos autores $(14,19)$ plantean que las culturas organizacionales saludables contribuyen a la retención de enfermeras, generan buenos resultados en el outcome del paciente y beneficios para el hospital. Las propias enfermeras piensan que tener pares competentes y apoyo para capacitación, son los elementos más importantes de un ambiente saludable y las enfermeras con certificación de especialistas manifiestan mayor satisfacción laboral que aquellas que no las tienen (20). En la misma línea, Heath et al. (21) señalan que un ambiente "magnético" cuyas características son el reconocimiento y valoración de las emociones, mantención de procesos estandarizados, favorecer el empoderamiento, los liderazgos fuertes, el sentido de comunidad y tener conocimiento claro de la misión y visión, también son elementos que favorecen el ambiente laboral. Respecto a factores motivacionales extrínsecos como las remuneraciones y los beneficios, Morgan y Lynn (18) concluyeron que el foco exclusivo en estos factores no era suficiente para retener a las enfermeras y Spetz y Given (22) también observaron que el aumento del sueldo no constituía un factor importante para evitar el abandono de estos profesionales.

Según lo descrito, se pudo concluir que el abandono se podría medir a través de elementos relacionados con la satisfacción laboral y que son cuatro los elementos que permiten explorar las causas del abandono de la práctica clínica de enfermería, a saber: primero, el reconocimiento de la profesión por el entorno, es decir, aquella valoración que hace la organización, el equipo de salud y la sociedad de la enfermera; segundo, los factores organizacionales, es decir, las condiciones institucionales que determinan la organización del trabajo de la enfermera; tercero, el desarrollo profesional representado por el crecimiento profesional que logra la enfermera clínica en las instituciones de salud y cuarto, el desgaste emocional y stress que surge por el ejercicio de su rol.

Teniendo la necesidad de conocer la realidad en Chile, el presente estudio tuvo como objetivo determinar las variables asociadas al abandono de las enfermeras de la práctica clínica hospitalaria (PCH) en la Región Metropolitana y ajustar un modelo predictivo para estimar la probabilidad de que un profesional de enfermería abandone la PCH.

\section{MATERIAL Y MÉTODO}

Tipo de estudio: Investigación de abordaje cuantitativo, de diseño transversal y correlacional. 
Muestra: Se implementó un sistema de reclutamiento de enfermeras en la red asistencial a través de una carta enviada por correo electrónico a 1.000 enfermeras de la Región Metropolitana de Chile. A cada enfermera que recibió la convocatoria se le solicitó que reenviara la invitación y la encuesta a sus contactos. En la carta se explicaba el propósito del estudio y se indicaba que la participación era voluntaria, asegurando reserva de identidad, y que implicaba contestar el instrumento y devolverlo a los investigadores por el mismo medio, así se obtuvieron 239 respuestas. Los tamaños de las muestras se determinaron de acuerdo al tiempo definido para el reclutamiento, que fue de 12 meses. Se conformaron dos muestras por conveniencia y de sujetos tipo, una integrada por 164 enfermeras que habían abandonado la $\mathrm{PCH}$ y otra muestra de 75 enfermeras que se encontraban trabajando en la PCH.

Variables: El abandono de la práctica clínica hospitalaria se definió como variable dependiente y las razones de abandono de la práctica clínica hospitalaria como independientes.

Recolección de datos e instrumentos: Con el propósito de levantar las razones que tuvieron las enfermeras para abandonar la $\mathrm{PCH}$, se realizó un focus group con 12 enfermeras que habían abandonado la $\mathrm{PCH}$ para migrar a diversas actividades, algunas relacionadas con la enfermería y otras no. Con la información extraída del focus group y aquella obtenida de la revisión bibliográfica, se elaboró un instrumento utilizando una escala Likert con 54 afirmaciones que debían ser calificadas con puntuaciones de 1 a 4 según posibilidades de respuesta desde muy en desacuerdo hasta muy de acuerdo, respectivamente, complementadas con dos preguntas abiertas para recoger las razones que dicen tener las enfermeras para abandonar y cuáles para permanecer en la $\mathrm{PCH}$.

Control de calidad de los datos: Se realizó análisis de fiabilidad a través del Alpha de Cronbach para estimar la consistencia interna o confiabilidad del instrumento. El análisis de fiabilidad mostró una alta consistencia interna: Alpha de Cronbach de 0,932 ( $\mathrm{n}=$ 121) en las respuestas de quienes abandonaron y de quienes no lo habían hecho. La validez de contenido del instrumento se verificó por tres jueces expertos, quienes evaluaron si las afirmaciones del instrumento contenían el dominio esperado, es decir, las posibles causas de abandono de la PCH. La validez de criterio no se realizó ya que no se encontró otro instrumento de las mismas características. Para determinar la validez de constructo de la variable "abandono" se realizó un análisis factorial, estimándose apropiado debido a que las condiciones se cumplieron. Las características psicométricas del instrumento fueron muy satisfactorias. Las asociaciones entre todas las variables del instrumento fueron significativas y positivas, es decir, a medida que los puntajes de una variable aumentaban también lo hacían los puntajes de la otra. Esto permitió concluir que las afirmaciones median el mismo constructo. El análisis exploratorio dio cuenta de 16 subdimensiones contenidas en 5 dimensiones subyacentes al constructo abandono que explicaron el $71,25 \%$ de la variabilidad total de esta conducta. Lo anterior indica una concordancia con el contenido del instrumento, por lo que se puede considerar válido para medir el abandono.

Análisis de datos: Para rescatar las causas de abandono se consideraron aquellos ítems que obtuvieron sobre un $60 \%$ de mención como fuente de posibles causas de abandono en ambos grupos. Para el análisis descriptivo se utilizaron medidas de frecuencia y de tendencia central y dispersión. Para determinar si las distintas dimensiones estaban asociadas con el abandono se utilizó Chi-cuadrado de asociación en tablas de contingencia. Para este último análisis las respuestas se agruparon en dos categorías: satisfacción (agrupa 
las respuestas muy de acuerdo y de acuerdo) e insatisfacción (agrupa las categorías muy en desacuerdo y en desacuerdo). Se realizó un análisis comparativo entre los grupos de enfermeras que abandonaron y las que no habían abandonado a través de una prueba $T$ de Student para muestras independientes y finalmente el ajuste preliminar de un modelo logístico para predecir la probabilidad de abandono de las enfermeras. Los niveles de confianza de las pruebas realizadas oscilaron entre 95 y 99\%. Una vez realizado el análisis de asociación a través de análisis de correlación y pruebas Chi-cuadrado, y cuyos resultados apuntaron a establecer las variables predictoras que tienen un efecto significativo sobre la variable respuesta binaria (abandono o no), se ajustó un modelo para estos datos, que corresponde al Modelo Logístico. Este modelo permitió calcular la probabilidad de que una enfermera abandone la $\mathrm{PCH}$. Para encontrar tal probabilidad fue necesario ajustar el modelo, estimando los parámetros a través del Método de Máxima Verosimilitud, considerando aquellas variables independientes significativas. El ajuste realizado a través del paquete estadístico SPSS en su versión 20.0 consideró todas las dimensiones independientes y significativas en estudio.

\section{RESULTADOS}

Características de la muestra: Según la edad, los 164 profesionales que no trabajaban en PCH tenían, en promedio, 44,2 (DS 9,25) años de edad, superior al promedio de 36,1 (DS 10,03) años que tenían los 75 que trabajaban en la PCH. Al comparar ambos grupos en torno al total de años trabajados, se observó una mayor cantidad de años en el grupo de las que habían abandonado la PCH (promedio 15,92 DS: 9,58), en comparación con el grupo que aún trabajaba en PCH (promedio 9,06 DS: 8,32). Respecto al período en que los sujetos de la muestra obtuvieron su título, este fue entre los años 1969 y 2012.

Causas de abandono: En este análisis se consideraron aquellos ítems que obtuvieron sobre un $60 \%$ de mención como fuente de posibles causas de abandono en ambos grupos. Las enfermeras encuestadas coinciden que los aspectos presentados en la Tabla 1 son los que producen mayores posibilidades de abandono. Las remuneraciones y sistema de turno son los aspectos más recurrentes en la selección de factores relacionados con las causas de abandono. Los otros aspectos seleccionados, tales como desarrollo profesional y reconocimiento, son factores también relevantes aunque en menor frecuencia. En las respuestas a las preguntas abiertas del cuestionario se señalaron varias razones, tanto para hacer abandono de la PCH como para permanecer en ésta. La primera razón para hacer abandono de la PCH es la baja renta, seguida por el sistema de turnos, el exceso de carga de trabajo y por último la falta de reconocimiento (Tabla 2). A su vez las razones señaladas como causas de permanencia en la $\mathrm{PCH}$ son en primer lugar la vocación, en $2^{\circ}$ lugar la percepción de una mejor remuneración y en 3er lugar la búsqueda de desarrollo profesional (Tabla 3 ). Con respecto al abandono, el $68 \%$ de las razones se concentran en las variables: renta, sistema de turnos, exceso de carga de trabajo y falta de reconocimiento. La razón que más frecuentemente se citó en primer lugar es el sistema de turnos (65\% de las menciones de primera prioridad). La razón más mencionada sobre el total, sin considerar la prioridad asignada, es la baja renta $(22 \%)$, seguida por el sistema de turnos $(20 \%)$ (Tabla 2). Con respecto a las razones para permanecer en la PCH, el 52\% de las menciones se concentran en 3 variables: vocación, remuneración y desarrollo profesional. La razón más mencionada como primera prioridad es la vocación (56\%), seguida por la remuneración (44\%). Este orden se mantiene cuando se analiza la frecuencia de menciones de manera absoluta. Un 5\% de las 
razones mencionadas indican que existe un grupo de enfermeras que prefieren el sistema de turnos (Tabla 3). Al revisar los análisis estadísticos de ítems de insatisfacción (Tabla 1) y de preguntas abiertas (Tablas 2 y 3 ) es posible afirmar que los resultados de ambos análisis son coherentes entre sí. El supuesto detrás del primer análisis es que las causas de insatisfacción son a su vez causas de abandono. Este supuesto queda confirmado como cierto en el análisis de las respuestas a las preguntas abiertas, ya que las razones más importantes de abandono son las mismas que de insatisfacción. Los resultados anteriores se relacionan estrechamente con las apreciaciones obtenidas en el focus group, que señalan como causas más importantes de abandono: el sistema de turnos, las remuneraciones y la falta de reconocimiento. En las respuestas a las preguntas abiertas aparece en forma frecuente el factor sobrecarga de trabajo como causa de insatisfacción.

Variables asociadas a la condición de abandono: Los ítems que están significativamente relacionados con el abandono (Tabla 4) se agrupan en tres dimensiones: 1. Reconocimiento, representado por la remuneración, beneficios asociados al cargo y estatus social; 2. Sistema de turnos, representado por el impacto físico familiar y social; 3. Desarrollo profesional, representado por la relación entre perfeccionamiento y desarrollo profesional, planes de carrera y condiciones para crecer profesionalmente. De estos ítems sólo hay 5 que no presentan diferencias significativas al comparar ambos grupos (afirmaciones 33, 39, 40, 25 y 48). Entre los ítems asociados significativamente al abandono, el No 48, que conforma la dimensión sistema de turno desde la perspectiva del funcionamiento familiar, muestra una asociación más débil ( $p$ $=0,05)$. Muestran una estrecha asociación las variables relativas al reconocimiento y estatus profesional $(\mathrm{p} \leq 0,04)$ afirmaciones 2,3 , 4, 9. El ítem 33 relativo a liderazgo es el único con significación de la dimensión factor organizacional, subdimensión liderazgo. Las variables relativas al desarrollo profesional presentan una asociación significativa ( $\mathrm{p} \leq$ 0,044), ítems 25, 26, 39, 40 y 42.

Modelo de predicción del Abandono: Según los coeficientes ajustados, el modelo de regresión logística resultante es:

$$
\left.P(s)^{\prime}\right)=\frac{1}{1+e^{-[-2,903+0,089(\text { edad })+0,24(\text { organiza })-0,152(\text { equipo })-0,148(\text { turnos })+0,069(\text { desprof })]}}
$$

Así, un ejemplo de cómo aplicar el modelo preliminar, para una enfermera de 36 años de edad, con puntajes en las dimensiones Reconocimiento (Organizacional $=10$ puntos;
Equipo $=8$ puntos), Sistema de turnos $=15 \mathrm{y}$ Desarrollo profesional $=12$, la probabilidad de abandono es calculada como sigue:

$$
P(s i ́)=\frac{1}{1+e^{-[-2,903+0,089(36)+0,24(10)-0,152(8)-0,148(15)+0,069(12)]}}=\frac{1}{1+e^{-0,093}}=0,5232
$$

Luego, esta enfermera con esas características, tiene un 52,32\% de probabilidades de abandonar en algún momento la práctica clínica hospitalaria. 


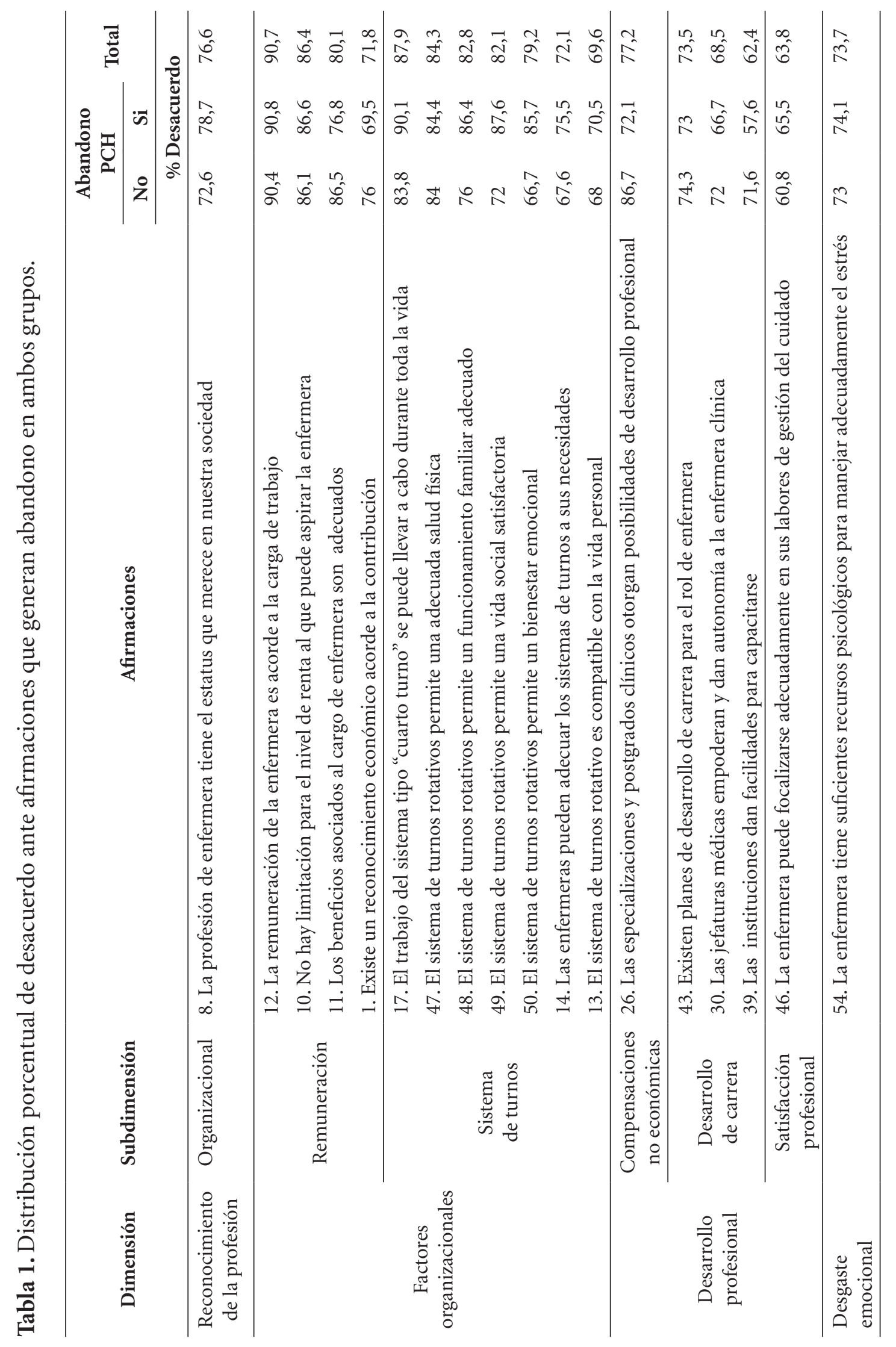


Tabla 2. Distribución porcentual de las razones de abandono en la PCH y menciones obtenidas.

\begin{tabular}{|c|c|c|c|c|c|c|c|c|}
\hline \multirow[t]{2}{*}{ Razones para abandonar } & \multirow[t]{2}{*}{$\mathrm{P} 1^{*}$} & \multirow[t]{2}{*}{$\mathrm{P}^{*}{ }^{\star}$} & \multirow[t]{2}{*}{$\mathrm{P}^{*}$} & \multirow[t]{2}{*}{$\begin{array}{c}\text { Total } \\
\text { Menciones }\end{array}$} & \multicolumn{3}{|c|}{$\begin{array}{l}\text { \% Sobre el total } \\
\text { de menciones } \\
\text { en cada razón }\end{array}$} & \multirow{2}{*}{$\begin{array}{l}\% \text { Sobre } \\
\text { el total de } \\
\text { menciones }\end{array}$} \\
\hline & & & & & $\% \mathrm{P} 1$ & $\% \mathrm{P} 2$ & $\% \mathrm{P3}$ & \\
\hline Baja renta (relativa a la carga de trabajo o absoluta) & 22 & 20 & 11 & 53 & $42 \%$ & $38 \%$ & $21 \%$ & $23 \%$ \\
\hline Sistema de turnos & 31 & 12 & 4 & 47 & $66 \%$ & $26 \%$ & $9 \%$ & $20 \%$ \\
\hline Exceso de carga de trabajo & 10 & 19 & 10 & 39 & $26 \%$ & $49 \%$ & $26 \%$ & $17 \%$ \\
\hline Falta de reconocimiento & 6 & 4 & 14 & 24 & $25 \%$ & $17 \%$ & $58 \%$ & $10 \%$ \\
\hline Escaso desarrollo profesional & 2 & 7 & 10 & 19 & $11 \%$ & $37 \%$ & $53 \%$ & $8 \%$ \\
\hline Mal ambiente laboral & 3 & 10 & 6 & 19 & $16 \%$ & $53 \%$ & $32 \%$ & $8 \%$ \\
\hline Entorpece vida familiar/social & 5 & 3 & 6 & 14 & $36 \%$ & $21 \%$ & $43 \%$ & $6 \%$ \\
\hline Stress & 0 & 3 & 4 & 7 & $0 \%$ & $43 \%$ & $57 \%$ & $3 \%$ \\
\hline Falta de espíritu de servicio/vocación & 2 & 3 & 2 & 7 & $29 \%$ & $43 \%$ & $29 \%$ & $3 \%$ \\
\hline Falta de recursos & 0 & 2 & 1 & 3 & $0 \%$ & $67 \%$ & $33 \%$ & $1 \%$ \\
\hline Maltrato laboral & 2 & 1 & 0 & 3 & $67 \%$ & $33 \%$ & $0 \%$ & $1 \%$ \\
\hline Total & 81 & 84 & 68 & 235 & & & & \\
\hline
\end{tabular}

* Número respuestas con una mención (P1), con dos (P2) y con tres (P3).

Tabla 3. Distribución porcentual de las razones de permanencia en la $\mathrm{PCH}$.

\begin{tabular}{|c|c|c|c|c|c|c|c|c|}
\hline \multirow[t]{2}{*}{ Razones para permanecer } & \multirow[t]{2}{*}{$\mathrm{P}^{*}$} & \multirow[t]{2}{*}{$\mathrm{P} 2^{*}$} & \multirow[t]{2}{*}{$\mathbf{P} 3^{*}$} & \multirow[t]{2}{*}{$\begin{array}{c}\text { Total } \\
\text { Menciones }\end{array}$} & \multicolumn{3}{|c|}{$\begin{array}{l}\text { \% Sobre el total } \\
\text { de menciones } \\
\text { en cada razón }\end{array}$} & \multirow{2}{*}{$\begin{array}{c}\% \text { Sobre } \\
\text { el total de } \\
\text { menciones }\end{array}$} \\
\hline & & & & & $\%$ P1 & $\% \mathrm{P} 2$ & $\% \mathrm{P3}$ & \\
\hline Vocación & 37 & 21 & 8 & 66 & $56 \%$ & $32 \%$ & $12 \%$ & $28 \%$ \\
\hline Mejor remuneración & 16 & 8 & 12 & 36 & $44 \%$ & $22 \%$ & $33 \%$ & $15 \%$ \\
\hline Desarrollo profesional & 2 & 11 & 7 & 20 & $10 \%$ & $55 \%$ & $35 \%$ & $9 \%$ \\
\hline Gusto por el sistema de turnos & 4 & 3 & 4 & 11 & $36 \%$ & $27 \%$ & $36 \%$ & $5 \%$ \\
\hline Buen ambiente laboral & 1 & 3 & 7 & 11 & $9 \%$ & $27 \%$ & $64 \%$ & $5 \%$ \\
\hline Reconocimiento laboral & & 6 & 4 & 10 & $0 \%$ & $60 \%$ & $40 \%$ & $4 \%$ \\
\hline Temor al cambio & 3 & 3 & 2 & 8 & $38 \%$ & $38 \%$ & $25 \%$ & $3 \%$ \\
\hline Condiciones demográficas & 2 & 3 & 1 & 6 & $33 \%$ & $50 \%$ & $17 \%$ & $3 \%$ \\
\hline Mayor estabilidad laboral & 2 & & 4 & 6 & $33 \%$ & $0 \%$ & $67 \%$ & $3 \%$ \\
\hline Lo prefieren a las labores administrativas & 1 & 2 & 1 & 4 & $25 \%$ & $50 \%$ & $25 \%$ & $2 \%$ \\
\hline No ven otra alternativa & & 4 & & 4 & $0 \%$ & $100 \%$ & $0 \%$ & $2 \%$ \\
\hline Total & 68 & 64 & 50 & 182 & & & & \\
\hline
\end{tabular}

* Número respuestas con una mención (P1), con dos (P2) y con tres (P3). 
Tabla 4. Resumen afirmaciones asociadas a abandono y comparación entre grupos ( $p \leq 0,05$; $p \leq 0,01)$.

\begin{tabular}{|c|c|c|c|}
\hline \multirow[t]{2}{*}{ Dimensión } & \multirow[t]{2}{*}{ Afirmaciones } & $\begin{array}{c}\text { Asociación } \\
\text { con abandono }\end{array}$ & $\begin{array}{l}\text { Comparación } \\
\text { entre grupos }\end{array}$ \\
\hline & & Valor $\mathbf{p}$ & Valor $\mathbf{p}$ \\
\hline \multirow{5}{*}{$\begin{array}{l}\text { Desarrollo } \\
\text { Profesional }\end{array}$} & $\begin{array}{l}\text { 26. Las especializaciones y postgrados clínicos otorgan } \\
\text { posibilidades de desarrollo profesional reconocido a } \\
\text { través de aumento de renta u otros beneficios relevantes }\end{array}$ & 0,016 & 0,004 \\
\hline & $\begin{array}{l}\text { 42. La forma de recompensar un buen desempeño clíni- } \\
\text { co suele ser un ascenso en cargos administrativos }\end{array}$ & 0,01 & 0,005 \\
\hline & 39. Las instituciones dan facilidades para capacitarse & 0,044 & 0,063 \\
\hline & $\begin{array}{l}\text { 40. La capacitación que reciben las enfermeras es acorde } \\
\text { a sus necesidades }\end{array}$ & 0,022 & 0,122 \\
\hline & $\begin{array}{l}\text { 25. Las especializaciones y postgrados clínicos otorgan } \\
\text { posibilidades de desarrollo profesional en el ámbito clí- } \\
\text { nico }\end{array}$ & 0,031 & 0,206 \\
\hline \multirow{4}{*}{$\begin{array}{l}\text { Sistema } \\
\text { de turnos }\end{array}$} & $\begin{array}{l}\text { 49. El sistema de turnos rotativos permite una vida so- } \\
\text { cial satisfactoria }\end{array}$ & 0,005 & 0,03 \\
\hline & $\begin{array}{l}\text { 50. El sistema de turnos rotativos permite un bienestar } \\
\text { emocional }\end{array}$ & 0,001 & 0,015 \\
\hline & $\begin{array}{l}\text { 33. Las enfermeras jefes están abiertas a recibir críticas } \\
\text { de parte de sus enfermeras subalternas }\end{array}$ & 0,028 & 0,892 \\
\hline & $\begin{array}{l}\text { 48. El sistema de turnos rotativos permite un funciona- } \\
\text { miento familiar adecuado }\end{array}$ & 0,054 & 0,168 \\
\hline \multirow{4}{*}{ Reconocimiento } & $\begin{array}{l}\text { 2. Existe en la organización algún tipo de reconocimien- } \\
\text { to en beneficios especiales (tales como flexibilidad hora- } \\
\text { ria) para una enfermera con desempeño destacado }\end{array}$ & 0 & 0 \\
\hline & $\begin{array}{l}\text { 3. El trabajo que desempeña la enfermera es percibido } \\
\text { como importante y significativo por la institución }\end{array}$ & 0,003 & 0,002 \\
\hline & $\begin{array}{l}\text { 4. Las sugerencias de la enfermera son consideradas por } \\
\text { la administración de la institución }\end{array}$ & 0,004 & 0,002 \\
\hline & $\begin{array}{l}\text { 9. La institución le permite a la enfermera participar en } \\
\text { decisiones relevantes }\end{array}$ & 0,004 & 0 \\
\hline
\end{tabular}

\section{DISCUSIÓN Y CONCLUSIÓN}

Se encontró que la primera causa de abandono de la PCH es el esquema de "cuarto turno", que hace que el trabajo profesional sea poco compatible con la vida personal de las enfermeras. Secundariamente aparecen como motivos de abandono de la PCH las bajas remuneraciones y el escaso reconocimiento organizacional. En Chile existe el esquema de cuarto turno, lo que es bastante único en el mundo y se caracteriza por el siguiente patrón: 12 horas de trabajo diurno continuado, al siguiente día 12 horas de trabajo de noche continuado y a continuación dos días libres, lo que se repite indefinidamente. Se ha demostrado que los turnos de más de 12 horas diarias y que el trabajo de más de 40 horas semanales producen aumento significativo 
de la tendencia a cometer errores (23) y que el trabajo en cuarto turno interfiere con actividades domésticas y no domésticas (24).

Las remuneraciones no aparecen como un factor relevante de abandono, salvo para grupos muy específicos que se distinguen por las características de ser mujer y soltera (7). $\mathrm{Al}$ respecto, el que la baja remuneración esté descrita en estudios de la Región, como el factor más importante para cambiar de trabajo (25), coincide con los resultados del presente estudio, cuestión que puede ser reflejo de la realidad laboral del continente.

Respecto a la insatisfacción laboral, este estudio coincide con otros (26) al encontrar que es el principal factor asociado a la intención de abandonar el empleo o la profesión y también coincide con aquellos $(12,13,27-$ 30) que plantean que los factores extrínsecos, como la remuneración y los beneficios laborales, son menos relevantes como factores de retención que los intrínsecos, tales como el compromiso con la organización, con los compañeros de trabajo y con el paciente.

Las respuestas a las preguntas abiertas del instrumento dieron cuenta de variables que se relacionarían con la retención de la enfermera en la PCH. La vocación sería una de ellas, y esto coincide con estudios que indican que el compromiso con el paciente es un factor de retención (29, 31-33). Lo mismo ocurre con el desarrollo profesional, no solo al coincidir con estudios que han llegado a los mismos resultados (26) sino con otros que indican que las enfermeras con mayor formación profesional presentan una menor rotación $(9,10)$. Llama la atención que el esquema de turnos también aparece como un elemento de retención ( $5 \%$ de las menciones) y la remuneración como factor de abandono. Esto podría tener su explicación en que el esquema de cuarto turno permite el pluriempleo, condición que a su vez permite doblar la remuneración de un profesional. Sería interesante indagar más en profundidad esta situación y conocer si existe o no una asociación directa.
El modelo predictivo resultante puede constituir un aporte al management de instituciones de salud, dado que puede motivar a diseñar estrategias orientadas a prevenir el abandono de las enfermeras de la PCH, y con ello no solo mejorar la calidad de la enfermería clínica y el cuidado del paciente, sino también impactar en la calidad de vida de las propias enfermeras. Las limitaciones de este estudio se relacionan, en primer lugar, con el instrumento utilizado, el que se centró en conocer las causas del abandono de la PCH relacionadas con el trabajo, y no se incluyeron variables personales, sociales o demográficas. Esto podría estar dando cuenta del porcentaje de varianza no explicada por el instrumento. En segundo lugar, por razones de accesibilidad y baja tasa de respuestas, los tamaños de ambos grupos fueron diferentes, lo que supone un grado distinto de representatividad de cada grupo. Finalmente, el instrumento impidió, en algunos casos, un control directo sobre la completitud, consistencia y calidad de los datos. Lo anterior fue resuelto realizando una limpieza exhaustiva de la base de datos, con la consecuente disminución de las unidades de análisis en ambos grupos.

Se concluye entonces que las principales causas de abandono de las enfermeras de $\mathrm{PCH}$ son el sistema de turnos y las remuneraciones y los motivos por los cuales las enfermeras se mantienen trabajando en la PCH son la vocación profesional, las remuneraciones y el desarrollo profesional.

\section{REFERENCIAS}

1. OECD Health Data 2013. [Internet]. Paris: Organisation for Economic Co-operation and Development; 2013 [citado 21 diciembre 2013]. Country Notes. Disponible en: http://www.oecd.org/chile/ oecdhealthdata2013-countrynotes.htm

2. Buerhaus PI, Needleman J, Mattke S, Stewart M. Strengthening hospital nurs- 
ing. Health Aff [Internet]. 2002; 21(5): 123-32. [citado 22 diciembre 2013]. Disponible en: http://content.healthaffairs. org/content/21/5/123.full.pdf + html

3. Aiken LH, Clarke SP, Sloane DM, Sochalski J, Silber JH. Hospital nurse staffing and patient mortality, nurse burnout, and job dissatisfaction. JAMA. 2002; 288(16): 1987-93.

4. Estabrooks CA, Midodzi WK, Cummings GG, Ricker KL, Giovannetti P. The impact of hospital nursing characteristics on 30day mortality. Nurs Res. 2005; 54(2): 7484.

5. Rafferty AM, Clarke SP, Coles J, Ball J, James P, McKee M, et al. Outcomes of variation in hospital nurse staffing in english hospitals: Cross-sectional analysis of survey data and discharge records. Int J Nurs Stud. 2007; 44(2): 175-82.

6. Simon M, Müller BH, Hasselhorn HM. Leaving the organization or the profession-a multilevel analysis of nurses' intentions. J Adv Nurs. 2010; 66(3) :616-26.

7. Black L, Spetz J, Harrington C. Nurses working outside of nursing societal trend or workplace crisis? Policy Polit Nurs Pract. 2008; 9(3): 143-57.

8. Black L, Spetz J, Harrington C. Nurses who do not nurse: Factors that predict non-nursing work in the US registered nursing labor market. Nurs Econ. 2010; 28(4): 245.

9. De Gieter S, Hofmans J, Pepermans R. Revisiting the impact of job satisfaction and organizational commitment on nurse turnover intention: An individual differences analysis. Int J Nurs Stud. 2011; 48(12): 1562-9.

10. Wang L, Tao H, Ellenbecker CH, Liu X. Job satisfaction, occupational commitment and intent to stay among Chinese nurses: A cross-sectional questionnaire survey. J Adv Nurs. 2012; 68(3): 539-49.

11. Lin SY, Chiang HY, Chen IaL. Comparing nurses' intent to leave or stay: Differences of practice environment perceptions.
Nurs Health Sci. 2011; 13(4): 463-7.

12. Shacklock K, Brunetto Y. The intention to continue nursing: Work variables affecting three nurse generations in Australia. J Adv Nurs. 2012; 68(1): 36-46.

13. Reitz O, Anderson MA. An overview of job embeddedness. J Prof Nurs. 2011; 27(5): 320-7.

14. Liu C, Zhang L, Ye W, Zhu J, Cao J, Lu $\mathrm{X}$, et al. Job satisfaction and intention to leave: A questionnaire survey of hospital nurses in Shanghai of China. J Clin. Nurs. 2012; 21(1-2): 255-63.

15. Tourangeau AE, Cummings G, Cranley LA, Ferron EM, Harvey S. Determinants of hospital nurse intention to remain employed: Broadening our understanding. J Adv Nurs. 2010; 66(1): 22-32.

16. Ulrich BT, Lavandero R, Hart KA, Woods D, Leggett J, Taylor D. Critical care nurses' work environments: A baseline status report. Crit Care Nurse. 2006; 26(5): 46-57.

17. Choi SP, Pang SM, Cheung K, Wong TK. Stabilizing and destabilizing forces in the nursing work environment: A qualitative study on turnover intention. Int J Nurs Stud. 2011; 48(10): 1290-301.

18. Morgan JC, Lynn MR. Satisfaction in nursing in the context of shortage. J Nurs Manag. 2009; 17(3): 401-10.

19. Schmalenberg C, Kramer M. Essentials of a productive nurse work environment. Nurs Res. 2008; 57(1): 2-13.

20. Schmalenberg C, Kramer M. Clinical units with the healthiest work environments. Crit Care Nurse. 2008; 28(3): 6577.

21. Heath J, Johanson W, Blake N. Healthy work environments: A validation of the literature. J Nurs Adm. 2004; 34(11): 52430

22. Spetz J, Given R. The future of the nurse shortage: Will wage increases close the gap? Health Aff (Millwood). 2003; 22(6): 199-206.

23. Rogers AE, Hwang W, Scott LD, Aiken LH, Dinges DF. The working hours of 
hospital staff nurses and patient safety. Health Aff (Millwood). 2004; 23(4): 20212.

24. Córdova VM, Hevia JC, Figueroa MA. Trabajo en turnos en el sector de la salud chileno: Una comparación entre el sector público y privado. Cienc.Trab. 2006; 8(21): 147-50.

25. Palmer SP. Nurse retention and satisfaction in Ecuador: Implications for nursing administration. J Nurs Manag. 2014; 22 (1): 89-96.

26. De Milt DG, Fitzpatrick JJ, McNulty SR. Nurse practitioners' job satisfaction and intent to leave current positions, the nursing profession, and the nurse practitioner role as a direct care provider. J Am Acad Nurse Pract. 2011; 23(1): 42-50.

27. Chan ZC, Tam WS, Lung MK, Wong WY, Chau CW. A systematic literature review of nurse shortage and the intention to leave. J Nurs Manag. 2013; 21(4): 605-13.

28. Mrayyan MT. Nurse job satisfaction and retention: Comparing public to private hospitals in Jordan. J Nurs Manag. 2005; 13(1): 40-50.

29. Wang L, Tao H, Ellenbecker CH, Liu X. Job satisfaction, occupational commitment and intent to stay among Chinese nurses: A cross sectional questionnaire survey. J Adv Nurs. 2012; 68(3): 539-49.

30. Sawatzky JA, Enns CL. Exploring the key predictors of retention in emergency nurses. J Nurs Manag. 2012; 20(5): 696707.

31. AbuAlRub RF. Work and non-work social support and intent to stay at work among Jordanian hospital nurses. Int Nurs Rev. 2010; 57(2): 195-201.

32. Tourangeau AE, Cummings G, Cranley LA, Ferron EM, Harvey S. Determinants of hospital nurse intention to remain employed: Broadening our understanding. J Adv Nurs. 2010; 66(1): 22-32.

33. Lee YW, Dai YT, Park CG, McCreary LL. Predicting quality of work life on nurses' intention to leave. J Nurs Scholarsh. 2013; 45(2): 160-168. 